\title{
Sodium Glucose Co-Transporter 2 (SGLT2) Inhibitor Dapagliflozinstabilizes Diabetes-Induced Atherosclerotic Plaque Instability
}

\section{Yung-Chih Chen}

Baker Heart and Diabetes Institute https://orcid.org/0000-0002-5387-5818

Karen Jandeleit-Dahm ( $\nabla$ karin.jandeleit-dahm@monash.edu )

University of Melbourne

Karlheinz Peter

Baker Heart and Diabetes Institute

\section{Methodology}

Keywords: Animal model, Streptozotocin, Sodium glucose co-transporter 2 inhibitor, Plaque instability

Posted Date: June 7th, 2021

DOI: https://doi.org/10.21203/rs.3.rs-540987/v1

License: (c) (1) This work is licensed under a Creative Commons Attribution 4.0 International License.

Read Full License 


\title{
Sodium glucose co-transporter 2 (SGLT2) inhibitor dapagliflozin stabilizes diabetes-induced atherosclerotic plaque instability
}

\author{
Yung Chih Chen ${ }^{1,2,3}$, Karin Jandeleit-Dahm ${ }^{3,4,5^{*}}$, Karlheinz Peter ${ }^{1,2,3,6^{*}}$ \\ ${ }^{1}$ Baker Heart \& Diabetes Institute, Melbourne, Australia \\ ${ }^{2}$ Central Clinical School, Monash University, Melbourne, Australia \\ ${ }^{3}$ Department of Cardiomentabolic Health, University of Melbourne, Melbourne, Australia \\ ${ }^{4}$ Department of Diabetes, Monash University, Melbourne, Australia \\ ${ }^{5}$ German Diabetes Centre, Leibniz Centre for Diabetes Research at Heinrich Heine \\ University, Duesseldorf, Germany \\ ${ }^{6}$ Department of Cardiology, The Alfre Hospital, Melbourne, Australia \\ *equally contributing senior authors
}

Corresponding authors:

Karlheinz Peter, MD PhD

Atherothrombosis \& Vascular Biology,

Baker Heart and Diabetes Institute

PO Box 6492, St Kilda Road Central

Melbourne, VIC 8008, Australia

Phone: +613 8532 1490; Fax: +613 85321100

Email: karlheinz.peter@baker.edu.au

Karin Jandeleit-Dahm, MD, PhD

Department of Diabetes

Monash University

99 Commercial Road

Melbourne, VIC 3004, Australia

Phone: + 6139903 0008; Fax : +613 99053288

Email: karin.jandeleit-dahm@monash.edu 


\section{Abstract}

\section{Aims/hypothesis:}

Diabetes is known to accelerate the progression of atherosclerosis and increase plaque instability. However, there has been a lack of suitable animal models to study the effect of diabetes on plaque instability. We hypothesized that the tandem stenosis (TS) mouse model, which reflects plaque instability and rupture as seen in patients, can be applied to study the effects of diabetes and its respective therapeutic approaches on plaque instability/rupture.

\section{Methods:}

$\mathrm{ApoE}^{-/-}$mice at 7 weeks of age were injected with streptozotocin (STZ) for 5 consecutive days. 5 weeks after STZ injection, mice were surgically subjected to TS in the right carotid artery and fed with a high-fat diet for an additional 7 weeks. To validate this newly developed animal model, administration of the interventional drug dapagliflozin was provided via drinking water $(25 \mathrm{mg} / \mathrm{kg}) 3$ days after TS surgery.

\section{Results:}

Diabetic mice showed an increase in the size of unstable atherosclerotic plaques in the TS model. Plaque instability markers such as MCP-1, CD68, and necrotic core (NC) size were significantly increased. Mice treated with the sodium glucose co-transporter $2 \mathrm{i}$ (SGLT2i) dapagliflozin demonstrated attenuated glucose levels. Importantly, these mice demonstrated plaque stabilization with enhanced collagen accumulation, increased fibrosis, increased capto-lesion ratios, and significant upregulation of plaque NADPH oxidase 4 (NOX4) expression.

\section{Conclusions/interpretation:}

The TS mouse model in combination with the application of STZ represents a highly suitable and unique mouse model for studying plaque destabilization under diabetic conditions. Furthermore, for the first time, we provide evidence of plaque-stabilizing effects of SGLT2i. Our data also suggest that this newly developed mouse model is an attractive preclinical tool 
for testing anti-diabetic drugs for their highly sought-after potential to stabilize atherosclerotic plaques.

Keyword: Animal model, Streptozotocin; Sodium glucose co-transporter 2 inhibitor; Plaque instability. 


\section{Background}

Myocardial infarction (MI) is a major cause of mortality/morbidity in patients with diabetes mellitus and typically atherosclerosis is the underlying pathology [1]. Diabetes has been postulated to accelerate the development of atherosclerosis and increase plaque instability/vulnerability, ultimately causing plaque rupture and MI. Therapeutic approaches to stabilizing vulnerable plaques in diabetic patients are thus highly sought-after. Until recently there was no conclusive proof that any of the antidiabetic drugs provided cardiovascular protection. However, the advent of new antidiabetic agents, such as the sodium glucose cotransporter 2 inhibitor (SGLT2i) and GLP-1 receptor agonists, has substantially changed this scenario, adding certain antidiabetic drugs to the standard repertoire of cardiologists. This paradigm shift is based on several randomized, controlled clinical trials demonstrating that SGLT-2 inhibitors confer beneficial effects on cardiovascular outcomes in patients with type 2 diabetes mellitus [2]. There is clinical evidence that SGLT2i protects against heart failure; however, although postulated as a mechanism, there is no direct clinical proof of antiatherosclerotic and/or plaque-stabilizing effects of SGLT2i.

We have previously validated a unique mouse model of plaque instability/rupture by introducing a tandem stenosis (TS) to the carotid artery [3]. This TS model closely reflects plaque instability/rupture as seen in patients and has recently been successfully used to define the plaque-stabilizing effects of a myeloperoxidase inhibitor [4] and anti-CD47 antibodies [5]. We now systematically use this model to investigate whether diabetes promotes plaque instability and, most importantly, whether SGLT2i exhibits plaque-stabilizing effects in this newly developed animal model. 


\section{Methods}

Animal studies

$\mathrm{ApoE}^{-/-}$mice of C57/B16 background (Australia Resource Centre, Western Australia) at 7 weeks of age were injected with streptozotocin (STZ) at a dose of $55 \mathrm{mg} / \mathrm{kg}$ for 5 consecutive days. At 12 weeks of age, 5 weeks after STZ, mice were anesthetized intraperitoneally by a mixture of ketamine $(100 \mathrm{mg} / \mathrm{kg})$ and xylazine $(20 \mathrm{mg} / \mathrm{kg})$. An incision was made in the neck and the right common carotid artery was dissected from circumferential connective tissues. TS with $150 \mu \mathrm{m}$ outer diameters was introduced, with the distal point $1 \mathrm{~mm}$ from the carotid artery bifurcation and the proximal point $3 \mathrm{~mm}$ from the distal stenosis. The stenosis diameter was obtained by placing a 6-0 blue braided polyester fiber suture around the carotid artery together with a $150 \mu \mathrm{m}$ needle that was tied to it and later removed as described previously [3, 4]. Animals were sacrificed at 7 weeks after surgery.

\section{Histology and immunohistochemistry}

The left and right common carotid arteries and brachiocephalic trunk were sectioned as described previously [3]. Serial cryosections were obtained at $100 \mu \mathrm{m}$ intervals at $1,500-$ $2,000 \mu \mathrm{m}$ proximal to the proximal suture in the right common carotid artery and from the brachiocephalic trunk and stored at $-80{ }^{\circ} \mathrm{C}$ until staining was performed. Picrosirius red staining was performed to highlight collagen within the atherosclerotic plaque. Hematoxylin and eosin $(\mathrm{H} \& \mathrm{E})$ staining was used for necrotic core determination and atherosclerotic plaque burden. Oil red o staining was used for lipid content quantification in the plaque. Images were obtained using a light microscope (Olympus BX50). Sections were incubated with rat antimouse TER-119 biotin-labeled antibody (eBioscience 13-5921) 1:400; rat anti-mouse CD68 (AbD Serotech MCA1957GA) 1:100 or MOMA-2 (AbD Serotech MCA519G); rabbit 
polyclonal anti-NOX4 (Sigma-Aldrich ABC459) 1:100; and anti-monocyte chemoattractant protein-1 antibody (Abcam ab7202) 1:200 at $4{ }^{\circ} \mathrm{C}$ overnight. After washing steps and incubation in $\mathrm{ABC}$ reagent, $\mathrm{DAB}$ reagent was applied to each section. Conversion of the $\mathrm{DAB}$ substrate into a colored product was monitored and slides were immersed in $\mathrm{dH}_{2} \mathrm{O}$ to stop the reaction. Sections were dehydrated and covered with coverslips using DPX mounting media. Quantification of images was performed on Fiji Image J[6].

Statistical analyses

Quantitative results are expressed as mean \pm SEM. Comparisons of parameters among two groups were made by the unpaired Student's t-test or Mann-Whitney test. Comparisons of table statistics were made by Fisher's exact test. Comparisons of parameters among three groups were made by one-way ANOVA followed by post hoc analysis using the NewmanKeuls test. A value of $\mathrm{P}<0.05$ was considered to be statistically significant.

\section{Results}

We induced insulin-deficient diabetes using the beta-cell toxin STZ in $\mathrm{ApoE}^{-/-}$mice on a highfat diet who underwent TS surgery after confirming diabetes. The diabetic mice showed significantly elevated glucose and glycated hemoglobin levels (Figure 1A, 1B). Under diabetic conditions, the locally defined unstable atherosclerotic plaque area in the TS model showed a significant increase (Figure 1C). Importantly, several typical markers of plaque instability, such as the monocyte chemoattractant protein-1 (MCP-1; Figure 1D), macrophages (CD68; Figure 1E), and necrotic core size (Figure 1F), were significantly increased as quantified as a percentage of plaque area. As a direct proof of plaque instability, intraplaque hemorrhage (IH) increased from $52 \%$ of untreated mice to $81 \%$ in STZ-treated mice with diabetes (Figure 1G) and plaque rupture increased from $32 \%$ to $56 \%$, respectively (Figure $1 \mathrm{H}$ ) as measured in a total 
of 226 mice, whereby 116 were untreated mice with TS and 100 were STZ-treated mice with TS. These data confirm that diabetic conditions cause plaque instability and demonstrate the suitability of the TS mouse model for detecting diabetes-associated changes in plaque instability.

Next, we examined the potential of the SGLT2 inhibitor dapagliflozin to stabilize plaques. Repeated STZ protocol as previously described, dapagliflozin was given to $\mathrm{ApoE}^{-/-}$mice via drinking water 3 days after TS surgery. 2 weeks after the start of dapagliflozin treatment, STZinduced hyperglycemia was modestly attenuated (Figure 2). The effect on lowering glucose was sustained for another 6 weeks; however, normoglycemia was never achieved. We focused on investigating markers of plaque instability. Interestingly, we did not see changes in inflammatory markers such as the macrophage markers (CD68 and MOMA-2) or MCP-1 levels (data not shown). Although the strongest markers of plaque instability such as plaque rupture and $\mathrm{IH}$ (data not shown) were unchanged, early signs of plaque stabilization such as an overall reduction in lipid content (Figure 3A) and increases in collagen content and the cap-to-core ratio were significant in the dapagliflozin-treated group (Figure 3B, 3C). These data are of substantial translational relevance as the cap-to-core ratio is a particularly important clinical marker that reflects plaque stability and is closely linked to improved clinical outcomes in patients.

Increased formation of reactive oxygen species (ROS) is associated with plaque instability in diabetes. NADPH oxidases, particularly increased expression of NADPH oxidase1 (NOX1) and reduced expression of the vasculoprotective NOX4 isoform in advanced plaques, have been shown to contribute to plaque development and remodeling in diabetes [7]. Interestingly, while expression of pro-atherosclerotic NOX-1 (data not shown) was not upregulated, the 
expression of anti-atherosclerotic NOX-4 (Figure 3D) was upregulated in the dapagliflozin group. Therefore, the regulation of certain NOX isoforms by SGLT2i therapy represents a potential mechanistic explanation for the beneficial effect seen in patients treated with SGLT2i.

\section{Discussion}

SGLT2i has emerged as a new therapeutic target for lowering glucose. Several clinical trials have demonstrated the efficacy of the glucose lowering of SGLT-2 inhibitors (EMPA-REG OUTCOME, CANVAS, DECLARE-TIMI) in association with improved cardiovascular outcomes [8-10]. The EMPA-REG OUTCOME trial of empagliflozin demonstrated a reduction in cardiovascular outcomes and death from other causes [10]. Nevertheless, direct evidence that SGLT2i stabilizes atherosclerotic plaques is still lacking, partly due to the fact that animal models of atherosclerosis typically do not exhibit plaque instability and rupture [11]. Currently, only a few experimental studies have investigated the effect of SGLT2i in classical mouse models of atherosclerosis. Al-Sharea et al. treated LDLR $^{-/-}$mice on a high-fat diet and after STZ application with dapagliflozin and reported a reduction in peripheral monocyte count and a lower degree of atherosclerosis, postulating a change in plasma lipoproteins as the major cause [12]. In $\mathrm{ApoE}^{-/-}$mice on a high-fat diet, empagliflozin reduced blood glucose, aortic plaque area, insulin resistance, and inflammation[13]. These studies provide the first experimental indication that SGLT2i exhibits anti-atherosclerotic effects. Our studies go beyond and demonstrate the plaque-stabilizing capability of SGLT2i and suggest that changes in the pro-oxidant enzyme NOX4 are mechanistically involved in plaque stabilization.

\section{Conclusion}

In conclusion, the tandem stenosis mouse model in conjunction with STZ-induced diabetes reflects plaque destabilization as seen in patients with diabetes and importantly provides initial 
proof of the plaque-stabilizing effects of SGLT2i. Indeed, these effects on plaque stability could explain at least in part the reduction of cardiovascular events seen in diabetic patients treated with SGLT2i and as such bears direct translational relevance. Our data also establish the TS mouse model in combination with STZ-treatment as a preclinical tool for testing of antidiabetic drugs for highly sought-after plaque-stabilizing effects. 


\section{Declarations}

\section{Ethics approval and consent to participate}

All experimental protocols and animal experiments have been approved by the Animal Ethics Committees of the Alfred Research Alliance approved number (E/1838/2018/B).

\section{Consent for publication}

Not applicable

\section{Availability of data and materials}

The datasets used and/or analysed during the current study are available from the corresponding authors on reasonable request.

\section{Competing interests}

All authors declare no competing interests.

\section{Funding}

Yung-Chih Chen is supported by the Heart Foundation Future leader fellowship (No:102068). Karlheinz Peter is supported by an NHMRC L3 investigator fellowship. The work was partially supported by an NHMRC project grant (APP1163233, K Jandeleit-Dahm).

\section{Authors' contributions}

YCC collected and analysed and interpreted the data. YCC performed the histological examination of the atherosclerotic plaques. YCC, KP, KJD co-designed the animal experiment and discussed the data, and jointly wrote the manuscript. All authors have read and have approved the final manuscript.

\section{Acknowledgments}

Not applicable 


\section{Figures}
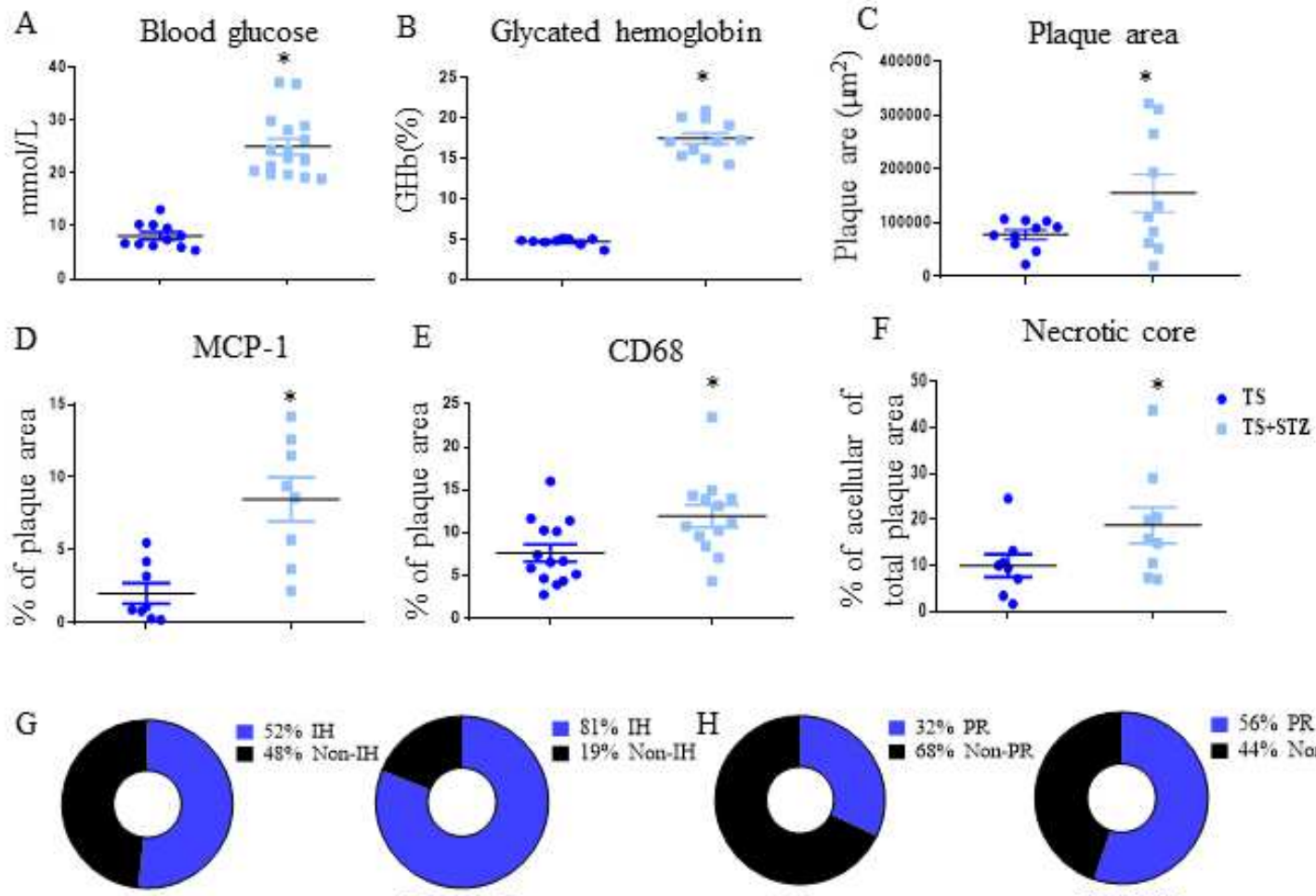

TS
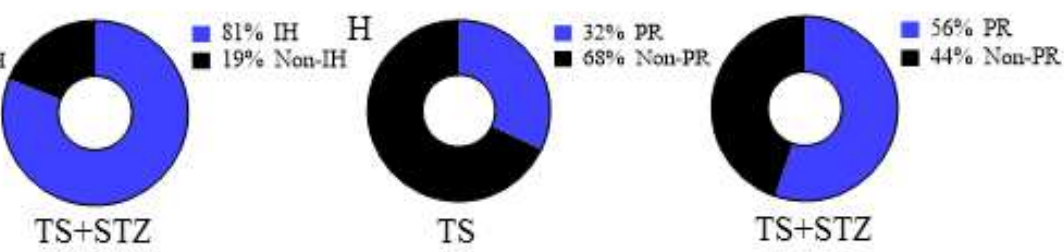

Figure 1: (A) The TS+STZ group shows upregulation of blood glucose levels compared to non-diabetic TS mice. (B) STZ-treated mice show an increase in the glycation of hemoglobin (GHb). Glycated hemoglobin A1c was measured by HPLC. (C) Diabetic mice show an increase in plaque area in the right carotid artery of the TS model. Quantification was performed on $H \& E$ stained sections, where the plaque was measured from the lumen to the internal elastic lamina. Each data point represents the mean of 6 sections per mouse at $200 \mu \mathrm{m}$ intervals. (D) MCP-1 is upregulated in diabetic mice with TS. The percentage of positive MCP-1 staining per atherosclerotic plaque area was obtained. Each data point represents the mean of 2 sections per mouse. (E) Unstable plaques in diabetic mice show an increase in macrophage staining. Macrophages were detected by staining for CD68 and quantified as described in D. (F) Unstable carotid plaques in diabetic mice show an increase in necrotic core size. The size of the NC is a major characteristic of plaque vulnerability in 
humans and we found that diabetes increases NC size. Quantification was performed as described in D. (G) Intraplaque hemorrhage (IH) is increased in diabetic mice. IH was defined by immunostaining for the red blood cell marker TER-119 or by visible hemorrhage. IH in the TS and TS+STZ groups were 60 out of 116 and 81 out of 100 , respectively. Non-IH in the TS and TS+STZ groups were 56 out of 116 and 19 out of 100 , respectively. $\mathrm{P}<0.0001$ by Fisher's exact test. (H) Plaque rupture (PR) was increased in diabetic mice. PR was defined by visible defects of the fibrous cap with or without luminal thrombi. PR in the TS and TS+STZ groups were 25 out of 96 and 48 out of 87 , respectively. Non-PR in the TS and TS+STZ groups were 71 out of 96 and 39 out of 87 , respectively. $\mathrm{P}<0.0001$ by Fisher's exact test. All other data are shown; $\mathrm{N}=13-16$, mean $\pm \mathrm{SEM}, * \mathrm{P}<0.05$ by Student t-test. 


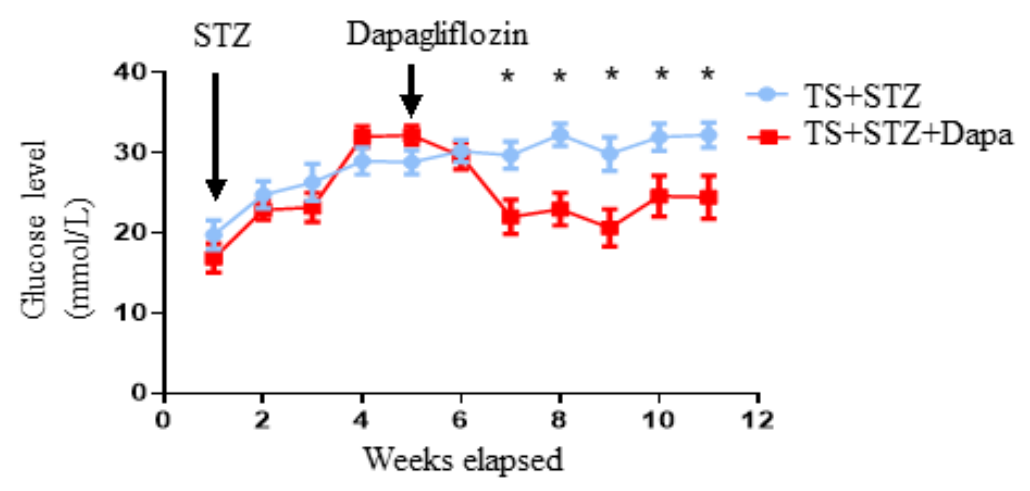

Figure 2: The SGLT2 inhibitor dapagliflozin reduces hyperglycemia in TS mice treated with STZ. Dapagliflozin was given via drinking water at $25 \mathrm{mg} / \mathrm{kg}$ after TS. 2 weeks after the initiation of daily dapagliflozin, glucose levels were significantly reduced to $24.66 \pm 1.43 \mathrm{mmol} / \mathrm{L}$ compared to vehicle-treated diabetic TS mice $28.61 \pm 1.13 \mathrm{mmol} / \mathrm{L}$ and remained steady until the endpoint. $\mathrm{N}=20-23$, mean $\pm \mathrm{SEM}, * \mathrm{P}<0.05$ by multiple $\mathrm{t}$-test. 

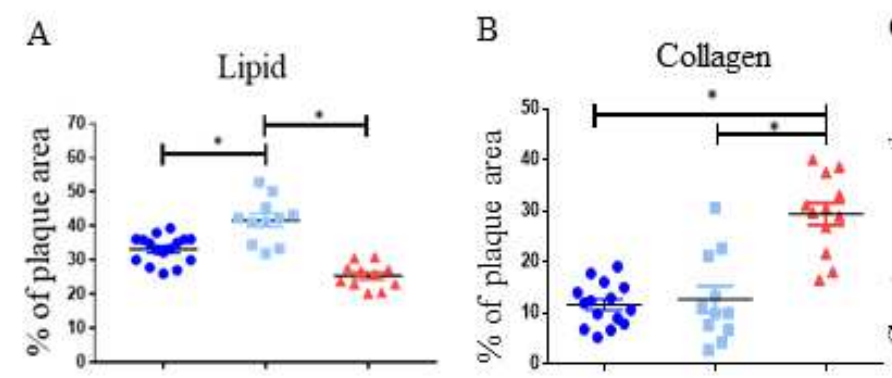

$\mathrm{C}$

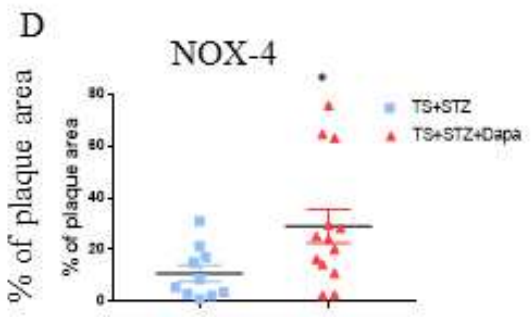

Figure 3: (A) Dapagliflozin significantly reduces the lipid content and (B) increases the collagen in the unstable atherosclerotic plaque area in the TS model. Oil Red O staining and Picrosirius red staining were used to quantify the total lipid and collagen content, respectively. (C) Diabetes significantly reduces the cap-to-core ratio, whereas dapagliflozin restores it, indicating a more stable plaque phenotype. The cap-to-core ratio was determined by dividing the total fibrous cap area by the maximal lesion height in every cross-section. $\mathrm{N}=13-16$, mean $\pm \mathrm{SEM}, * \mathrm{P}<0.05$ by ANOVA with Dunnett's multiple comparisons test. (D) Dapagliflozin increases NOX4 expression in unstable plaques of the TS model. The NOX4 expression in plaques was investigated by immunohistochemistry and each data point represents the mean of 2 sections per mouse. $\mathrm{N}=13-16$, mean $\pm \mathrm{SEM},{ }^{*} \mathrm{P}<0.05$ by Student's t-test. 


\section{References}

[1] Cavallari I, Bhatt DL, Steg PG, et al. (2021) Causes and Risk Factors for Death in Diabetes: A Competing-Risk Analysis From the SAVOR-TIMI 53 Trial. J Am Coll Cardiol 77(14): 1837-1840. 10.1016/j.jacc.2021.02.030

[2] Furtado RHM, Bonaca MP, Raz I, et al. (2019) Dapagliflozin and Cardiovascular Outcomes in Patients With Type 2 Diabetes Mellitus and Previous Myocardial Infarction. Circulation 139(22): 2516-2527. 10.1161/CIRCULATIONAHA.119.039996

[3] Chen YC, Bui AV, Diesch J, et al. (2013) A Novel Mouse Model of Atherosclerotic Plaque Instability for Drug Testing and Mechanistic/Therapeutic Discoveries Using Gene and MicroRNA Expression Profiling. Circ Res 113(3): 252-265.

10.1161/CIRCRESAHA.113.301562

[4] Rashid I, Maghzal GJ, Chen YC, et al. (2018) Myeloperoxidase is a potential molecular imaging and therapeutic target for the identification and stabilization of high-risk atherosclerotic plaque. Eur Heart J 39(35): 3301-3310. 10.1093/eurheartj/ehy419

[5] Kojima Y, Volkmer JP, McKenna K, et al. (2016) CD47-blocking antibodies restore phagocytosis and prevent atherosclerosis. Nature 536(7614): 86-90. 10.1038/nature18935

[6] Schneider CA, Rasband WS, Eliceiri KW (2012) NIH Image to ImageJ: 25 years of image analysis. Nat Methods 9(7): 671-675. 10.1038/nmeth.2089

[7] Gray SP, Di Marco E, Kennedy K, et al. (2016) Reactive Oxygen Species Can Provide Atheroprotection via NOX4-Dependent Inhibition of Inflammation and Vascular Remodeling. Arterioscler Thromb Vasc Biol 36(2): 295-307.

10.1161/ATVBAHA.115.307012

[8] Wiviott SD, Raz I, Bonaca MP, et al. (2019) Dapagliflozin and Cardiovascular Outcomes in Type 2 Diabetes. N Engl J Med 380(4): 347-357. 10.1056/NEJMoa1812389

[9] Neal B, Perkovic V, Mahaffey KW, et al. (2017) Canagliflozin and Cardiovascular and Renal Events in Type 2 Diabetes. N Engl J Med 377(7): 644-657.

10.1056/NEJMoa1611925

[10] Zinman B, Wanner C, Lachin JM, et al. (2015) Empagliflozin, Cardiovascular Outcomes, and Mortality in Type 2 Diabetes. N Engl J Med 373(22): 2117-2128.

10.1056/NEJMoa1504720

[11] Getz GS, Reardon CA (2012) Animal models of atherosclerosis. Arterioscler Thromb Vasc Biol 32(5): 1104-1115. 10.1161/ATVBAHA.111.237693

[12] Al-Sharea A, Murphy AJ, Huggins LA, Hu Y, Goldberg IJ, Nagareddy PR (2018)

SGLT2 inhibition reduces atherosclerosis by enhancing lipoprotein clearance in Ldlr(-/-) type 1 diabetic mice. Atherosclerosis 271: 166-176. 10.1016/j.atherosclerosis.2018.02.028

[13] Han JH, Oh TJ, Lee G, et al. (2017) The beneficial effects of empagliflozin, an SGLT2 inhibitor, on atherosclerosis in ApoE (-/-) mice fed a western diet. Diabetologia 60(2): 364-376. 10.1007/s00125-016-4158-2 


\section{Figures}
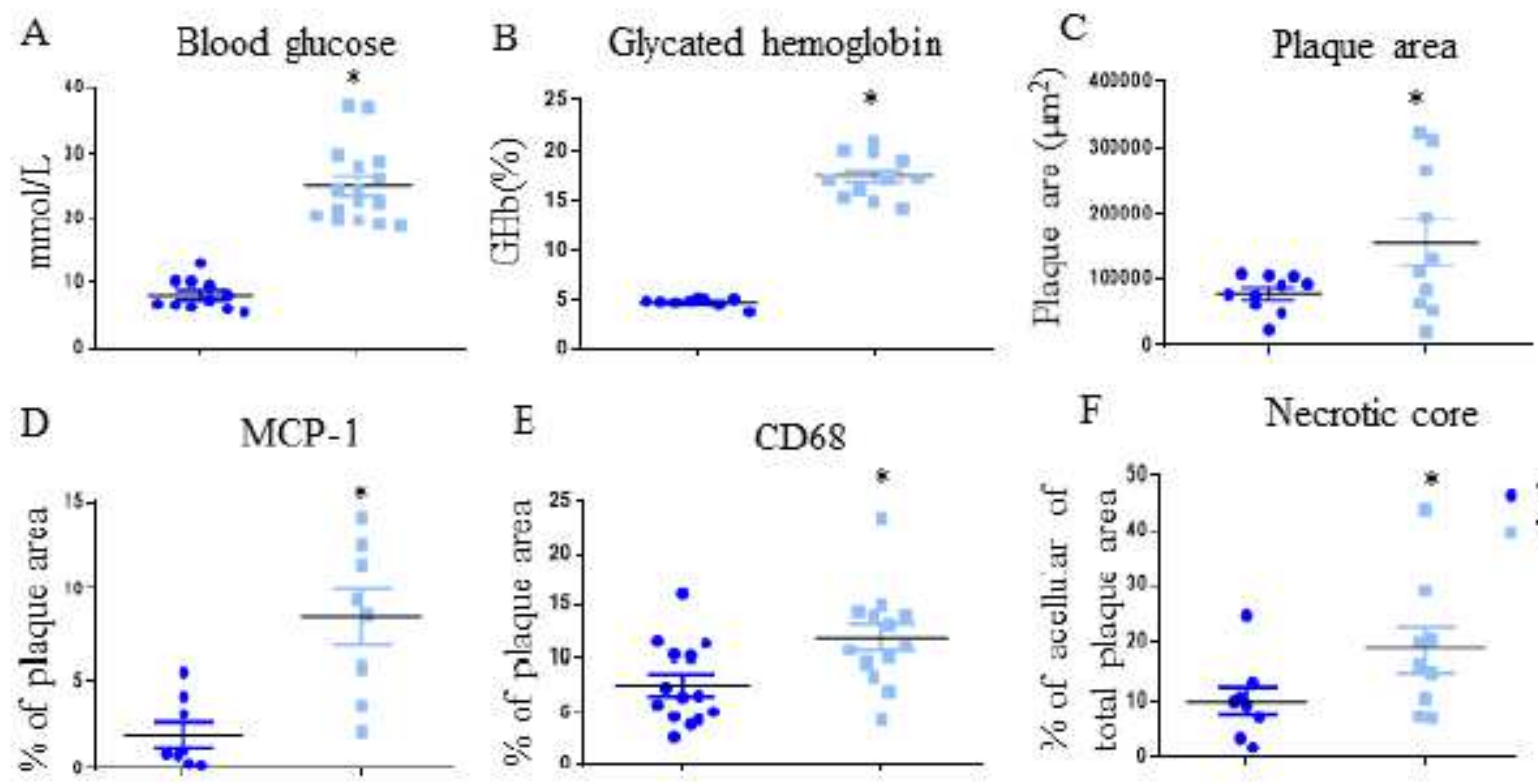

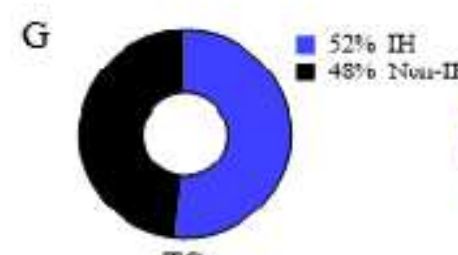

TS

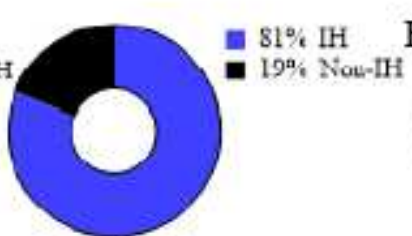

TS+STZ

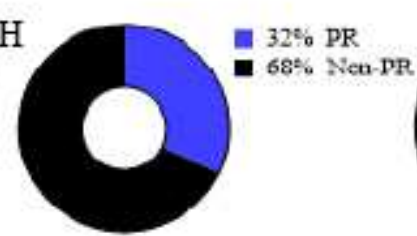

TS

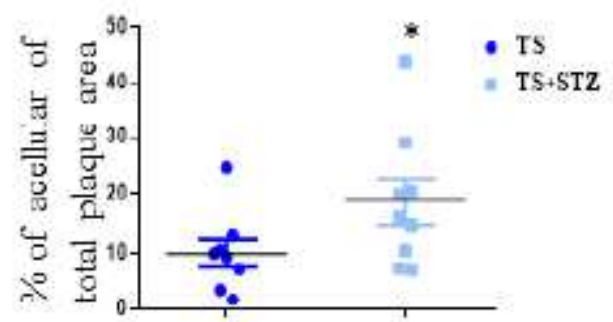

\section{Figure 1}

(A) The TS+STZ group shows upregulation of blood glucose levels compared to non-diabetic TS mice. (B) STZ-treated mice show an increase in the glycation of hemoglobin (GHb). Glycated hemoglobin A1c was measured by HPLC. (C) Diabetic mice show an increase in plaque area in the right carotid artery of the TS model. Quantification was performed on H\&E stained sections, where the plaque was measured from the lumen to the internal elastic lamina. Each data point represents the mean of 6 sections per mouse at $200 \mu \mathrm{m}$ intervals. (D) MCP-1 is upregulated in diabetic mice with TS. The percentage of positive MCP-1 staining per atherosclerotic plaque area was obtained. Each data point represents the mean of 2 sections per mouse. (E) Unstable plaques in diabetic mice show an increase in macrophage staining. Macrophages were detected by staining for CD68 and quantified as described in D. (F) Unstable carotid plaques in diabetic mice show an increase in necrotic core size. The size of the NC is a major characteristic of plaque vulnerability in humans and we found that diabetes increases NC size. Quantification was performed as described in D. (G) Intraplaque hemorrhage (IH) is increased in diabetic mice. IH was defined by immunostaining for the red blood cell marker TER-119 or by visible hemorrhage. $\mathrm{IH}$ in the TS and TS+STZ groups were 60 out of 116 and 81 out of 100, respectively. Non-IH in the TS and TS+STZ groups were 56 out of 116 and 19 out of 100 , respectively. $P<0.0001$ by Fisher's exact test. $(H)$ 
Plaque rupture (PR) was increased in diabetic mice. PR was defined by visible defects of the fibrous cap with or without luminal thrombi. PR in the TS and TS+STZ groups were 25 out of 96 and 48 out of 87, respectively. Non-PR in the TS and TS+STZ groups were 71 out of 96 and 39 out of 87, respectively. $P<0.0001$ by Fisher's exact test. All other data are shown; $N=13-16$, mean $\pm S E M, * P<0.05$ by Student $t-$ test.

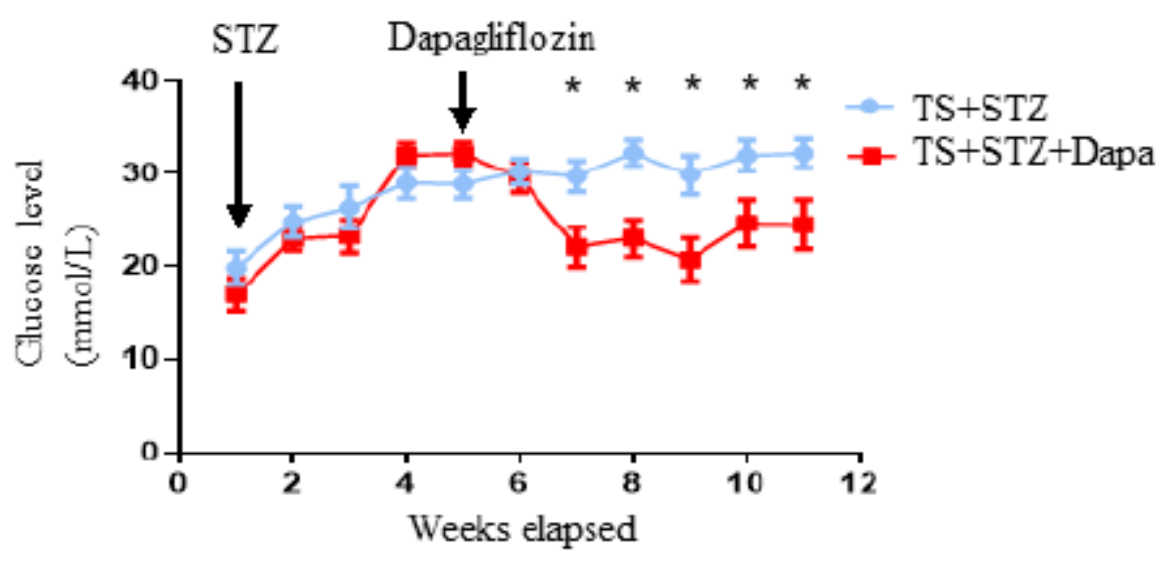

Figure 2

The SGLT2 inhibitor dapagliflozin reduces hyperglycemia in TS mice treated with STZ. Dapagliflozin was given via drinking water at $25 \mathrm{mg} / \mathrm{kg}$ after TS. 2 weeks after the initiation of daily dapagliflozin, glucose levels were significantly reduced to $24.66 \pm 1.43 \mathrm{mmol} / \mathrm{L}$ compared to vehicle-treated diabetic TS mice $28.61 \pm 1.13 \mathrm{mmol} / \mathrm{L}$ and remained steady until the endpoint. $\mathrm{N}=20-23$, mean $\pm S E M, * P<0.05$ by multiple $\mathrm{t}-$ test.

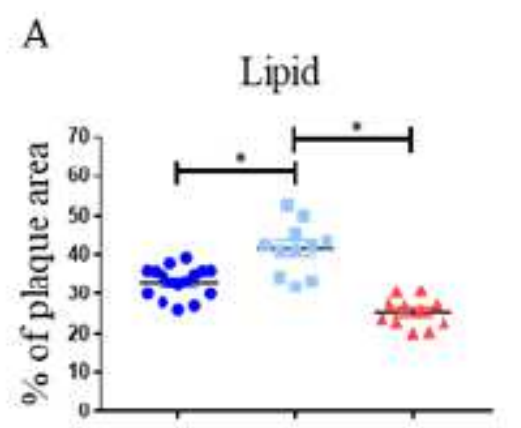

B

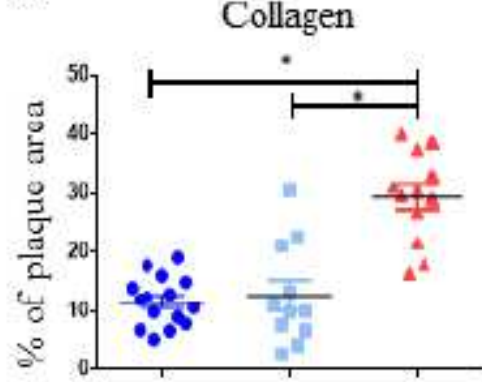

C Cap to core ratio

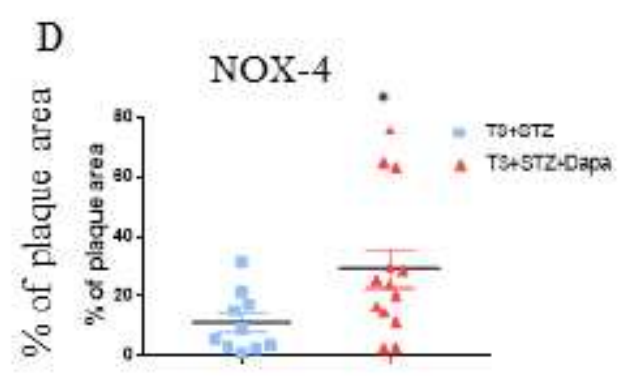

Figure 3 
(A) Dapagliflozin significantly reduces the lipid content and (B) increases the collagen in the unstable atherosclerotic plaque area in the TS model. Oil Red $\mathrm{O}$ staining and Picrosirius red staining were used to quantify the total lipid and collagen content, respectively. (C) Diabetes significantly reduces the cap-tocore ratio, whereas dapagliflozin restores it, indicating a more stable plaque phenotype. The cap-to-core ratio was determined by dividing the total fibrous cap area by the maximal lesion height in every crosssection. $N=13-16$, mean $\pm S E M,{ }^{*} P<0.05$ by ANOVA with Dunnett's multiple comparisons test. (D) Dapagliflozin increases NOX4 expression in unstable plaques of the TS model. The NOX4 expression in plaques was investigated by immunohistochemistry and each data point represents the mean of 2 sections per mouse. $\mathrm{N}=13-16$, mean $\pm S E M,{ }^{*} \mathrm{P}<0.05$ by Student's t-test. 